\title{
Utilization of the Terrestrial Weed Guduchi (Tinospora cordifolia) in Clean-Green Synthesis of Gold Nanoparticles
}

\author{
Tasneem Abbasi ${ }^{2}$, J Anuradha ${ }^{1}$ and SA Abbasi ${ }^{1 *}$ \\ ${ }^{1}$ Centre for Pollution Control and Environmental Engineering, Pondicherry University, Puducherry, India \\ ${ }^{2}$ Concurrently Visiting Associate Professor, Department of Fire Protection Engineering, Worcester, Polytechnic Institute, Worcester, MA 01609, USA
}

Received: July 29, 2014; Accepted: November 06, 2014; Published: December 05, 2014

*Corresponding author: SA Abbasi, Centre for Pollution Control and Environmental Engineering, Pondicherry University, Kalapet, Puducherry-605014, India, Tel: 0413-2654398; E-mail: s.a.abbasi@gmail.com

\begin{abstract}
A biomimetic method of gold nanoparticle (AuNP) synthesis is presented which utilizes a terrestrial weed guduchi (Tinospora cordifolia), as the main bioagent. The method enables rapid synthesis of AuNPs at ambient temperature and pressure, with frugal energy use and no harmful emissions. It is possible to control the shape and size of the product by controlling the metal-bioagent stoichiometry. The electron micrographs of the synthesized AuNPs reveal the presence of particles of either monodispersed spherical or polydispersed triangular, pentagonal, and hexagonal shapes in sizes of 16-30 $\mathrm{nm}$ and $25-75 \mathrm{~nm}$ respectively. The presence of gold atoms was confirmed from the EDAX and X-ray diffraction studies. FT-IR spectral study indicated that the alkaloids in the plant extract could have been responsible for the reduction-cum-stabilization of the gold ions into AuNPs.
\end{abstract}

\section{Introduction}

Advancements in nanotechnology are essentially driven by the exploration of newer nanoparticles which can play highly specialized roles in imaging, drug delivery, artificial implants, diagnostics, tissue engineering and gene delivery [13]. Gold nanoparticles (AuNPs), have been distinguished by their biocompatibility and unique structural, electronic, magnetic, optical and catalytic properties [4]. Due to this, the synthesis of nanoparticles in general, and AuNPs in particular, in an economical and eco-friendly manner has become an important thrust area of nanotechnology [5,6].

For long nanoparticles were manufactured by physical or chemical methods. Of these the physical route is highly energyintensive while the chemical methods often involve hazardous process conditions (high temperature and /or pressure), or release toxic byproducts. In contrast, the more recently introduced $[7,8]$ biomimetic methods try to extra-cellularly accomplish what microorganisms, algae, or plants do in nature viz synthesizing nanoparticles at ambient temperature and pressure in an ecologically benign manner. Among the organisms explored in the biomiming route, plants have proved most convenient and inexpensive [9]. Their aqueous extracts have been found to provide the biomolecules that are needed to reduce metal ions into atomic form and then stabilize the aggregating atoms before they grow beyond nano-size [6,7]. Similar role is played by the biomolecules that are contained in microorganisms but it is much more difficult (and expensive) to maintain microbial cultures and access their biomolecules than is possible with plants [10].

Whereas a number of reports exist on the use of medicinal, cosmetic, ornamental, or food/feed plants for synthesizing AuNPs, very few reports exist in which weedy plants have been utilized [11,12]. The species studied so far mostly encompass fruits, flowers, vegetables, grains, cereals, spices, other foodstuff, medicinal plants, and beauty aids. For example, geranium, neem, gooseberry, aloe vera, coriander, guava, clove buds, mint, cinnamon, curry leave, horse gram, myrobalan, white gourd and citrus fruit that already have well-established uses, and entail substantial costs of production, have been explored $[6,13,14]$. Also, in the past most authors have used only one or the other part of the plants-leaf, bark, seed, flower, or fruit-for AuNP synthesis. In contrast the present study is based on the use of whole plant of a weed. The weed guduchi, (Tinospora cordifolia), also called amritha, is a perennial climber, belonging to the Menispermaceae family. It has been valued for its medicinal properties in the classical Indian systems of medicine but is rarely used at present. By now, it has become weedy in several parts of south India [15] harming biodiversity and calling for measures to control it by finding ways for its utilization. It has been explored for the synthesis of silver nanoparticles [16,17], but there is no previous report on its use in synthesis AuNPs.

\section{Experimental}

All chemicals were of analytical reagent grade unless stated otherwise. Deionized and double distilled water was used for all analytical work.

\section{Preparation of the guduchi extract}

T. cordifolia was collected from its natural habitat near Pondicherry University, Puducherry. The fresh, mature, and disease-free plant portions were washed thoroughly with water, and then dipped in saline water to sterilize their surface, followed by washing them liberal and wiping 
them free of adhering water. A known quantity of pooled plant samples was dried at $105^{\circ} \mathrm{C}$ to a constant weight [18]. On the basis of dry weight thus obtained, extracts for nanoparticle synthesis were made by boiling $2 \mathrm{~g}$ dry weight equivalent of the plant material with $100 \mathrm{ml}$ of water for 5 minutes. The contents were filtered through a Whatman no. 42 filter paper and the filtrate was stored under refrigeration at $4^{\circ} \mathrm{C}$ [9]. Reconnoitery experiments indicated that the extracts retained their integrity for up to 3 days, as evidenced by the extent of intensity of nanoparticles generated by them. Hence in all the experiments the extracts were used within 3 days of preparation.

\section{Au (III) solution}

A $10^{-3} \mathrm{M}$ stock solution of $\mathrm{Au}(\mathrm{III})$ was prepared in water and was stored in amber bottles covered with black sheets.

\section{Nanoparticle synthesis}

The process involved mixing of plant extracts with $A u$ (III) solution in different stoichiometric ratios at ambient temperature and pressure. No stirring was necessary. The gold nanoparticles (AuNPs) began forming almost immediately as indicated by the appearance of pinkish red or purple color which grew in intensity with time. The color and its intensity depends on the stoichiometric ratio in which the plant extract and the metal ion had been mixed. The progress of the synthesis was monitored and quantified by recoding the spectra of the reaction mixtures using UV-visible spectrophotometer.

\section{Characterization of the synthesized AuNPs}

The UV-visible spectra were recorded with Labindia UV $3000^{+}$and ELICO SL 164 double beam instruments operated at $1 \mathrm{~cm}$ light-path length and $\pm 1 \mathrm{~nm}$ wavelength resolution.

For SEM (Scanning Electron Microscopy) and TEM (Transmission Electron Microscopy) studies the AuNPs were centrifuged at 12,000 rpm for 20 minutes using Remi C 24 centrifuge. The resulting pellets were washed thrice with water to remove the unreacted constituents and were re-dispersed in water.

The samples were readied for SEM by placing a drop of the AuNPs on a carbon-coated SEM grid. For High Resolution SEM (HRSEM), the samples were prepared by placing dried pellets on a carbon coated aluminium stub. For TEM the AuNPs were first pelletized by diluting and through sonication. The micrographs were recorded by depositing a drop of the well-dispersed samples on carbon-coated 300 mesh placed on copper TEM grids while the excess liquid was wiped off with filter paper.

The energy dispersive X-ray (EDAX) spectrum was recorded after documenting the electron micrographs in the spot-profile mode by focusing on the densely occupied gold nanoparticle region.

The Selected Area Electron Diffraction (SAED) pattern was recorded on an accessory that was equipped with the TEM instrument.

For the XRD (X-ray diffraction) spectra, the AuNP samples were prepared by drop-coating the pelletized AuNPs on a glass slide and scanning in the $2 \theta$ region, from $30^{\circ}$ to $80^{\circ}$, at $0.02^{\circ}$ per minute, and with the time constant of 2 seconds. The crystalline pattern of the nanoparticles was recorded using $\mathrm{Cu} \mathrm{K}_{\alpha 1}$ radiation with a wavelength $(\lambda)$ of $1.5406 \AA$ at a tube voltage of $40 \mathrm{kV}$ and a tube current of $30 \mathrm{~mA}$.

The Fourier Transform Infrared Spectroscopic (FTIR) studies were carried out on samples which were dried completely and were ground with potassium bromide. The spectra were recorded at diffuse mode with $4 \mathrm{~cm}^{-1}$ resolution in the mid-IR region between 4000 and $400 \mathrm{~cm}^{-1}$.

\section{Results and Discussion}

\section{UV-visible spectra}

Figures 1-4 present the UV-vis spectral patterns recorded from the AuNPs generated from the mixing of the aqueous plant broth and chloroauric acid solution. The bioreduction of the gold ions and their stabilization as nanosized atomic aggregates is seen to commence within $10 \mathrm{~min}$ of the start of the reaction. It is manifested by the gradual appearance of pinkish red or violet color in the reaction medium. This color has been known to be caused due to the excitation of surface Plasmon oscillations in the AuNPs induced by the passing light $[6,9,13,19]$.

Based on the proportions of stem extract with respect to the metal solution, two types of spectra were obtained one with a single peak in the visible region, and the other with double peak; the primary peak in the visible region and the secondary peak in the NIR (near infra-red) region. The reaction combination $\mathrm{SE}_{1}$ (Figure 1 and 2) exhibited the former type of spectra with $\lambda_{\max }$ ca. $551 \mathrm{~nm}$; while the $\mathrm{SE}_{2}$ (Figure 3 and 4) combinations exhibited the latter type of spectra with $\lambda_{\max }$ at ca. 555 and $980 \mathrm{~nm}$. The absorbance increased gradually to saturation with increase in the incubation period. The presence of a single peak in the visible region in the spectra of $\mathrm{SE}_{1}$ reaction combinations indicated that it might have occurred due to the Transverse (out-of-plane) Plasmon Resonance (TPR), which is exhibited by spherical nanoparticles. The SEM and TEM micrographs confirmed the presence of spherical particles. The spectral pattern of $\mathrm{SE}_{2}$ combinations shows the presence of two prominent absorption bands-a lower wavelength transverse absorption band in the visible region (out-of-plane vibration band) and a longer

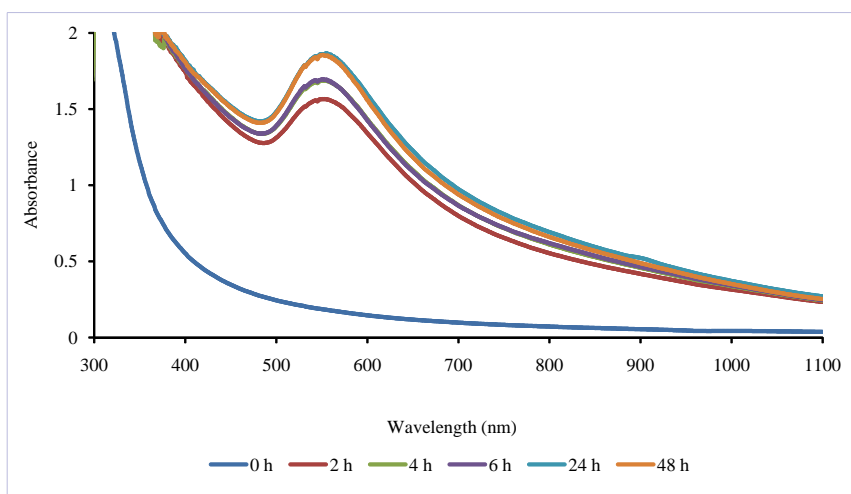

Figure 1: UV-visible spectra of $\mathrm{SE}_{1}$ metal-guduchi combination. 


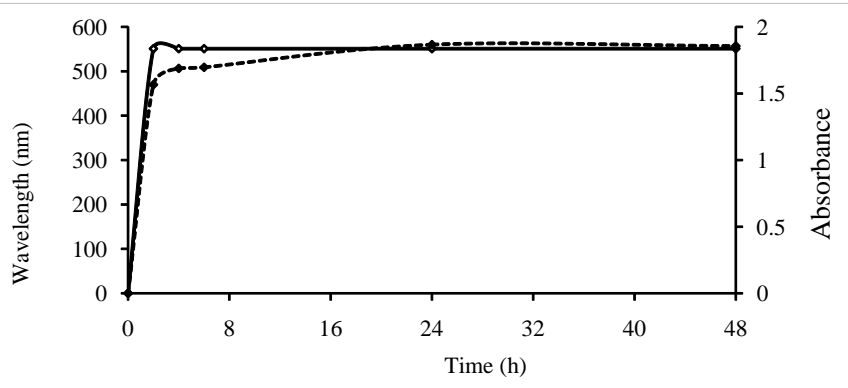

$\longrightarrow$ Peak wavelength $(\mathrm{nm}) \quad \cdots$ A bsorbance

Figure 2: Intensity of surface Plasmon resonances peak of $\mathrm{SE}_{1}$ as a function of the reaction duration.

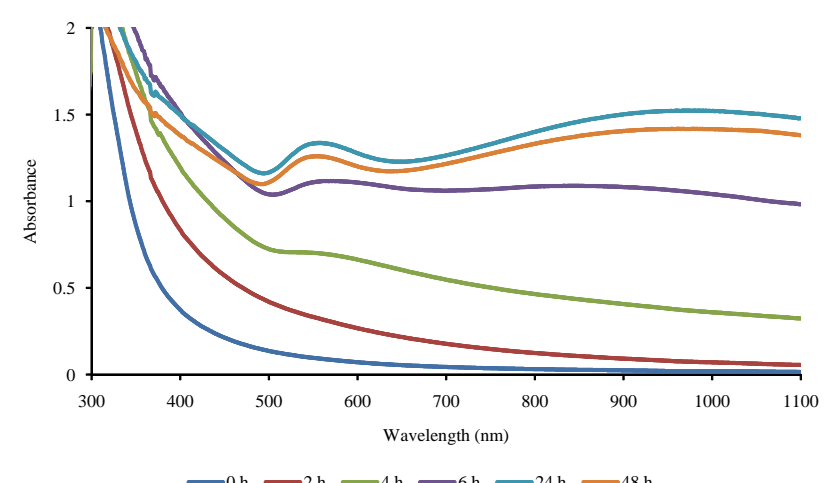

Figure 3: UV-visible spectra of $\mathrm{SE}_{2}$ metal-guduchi combination.

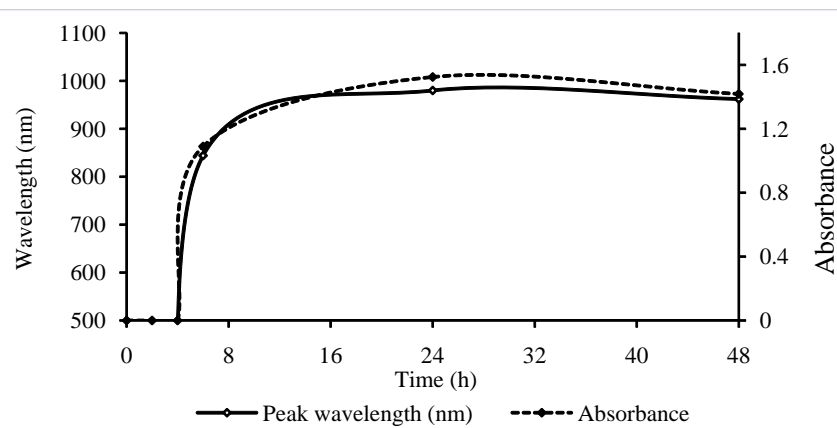

Figure 4: Intensity of the surface plasmon resonance peaks of $\mathrm{SE}_{2}$ as a function of the reaction duration.

wavelength longitudinal absorption band in the NIR region (inplane plasmon vibrations) - indicating that the synthesized nanoparticles possessed an intrinsic anisotropy [8,20,21] (Table 1). This was also confirmed from the SEM and TEM studies. Table 2 presents the summary of results obtained from the spectrophotometric studies for all the reaction combinations.

The AuNP formation was found to be almost complete within $24 \mathrm{~h}$. The synthesized AuNPs were characterized for their size, shape, purity and crystal structure by employing SEM, Hr-SEM, TEM, EDX, XRD and SAED techniques. The functional biomolecules involved in the reduction and stabilization process was identified using FT-IR spectroscopy.

\section{Electron microscopic (SEM, Hr-SEM, TEM) and EDX studies}

As may be seen from figure 5, which shows the SEM and $\mathrm{Hr}$ SEM micrographs recorded for the GNPs synthesized from the $\mathrm{SE}_{1}$ combination; the GNPs were of spherical shape. The TEM micrographs for the same GNPs mixture shows that the size of the nanoparticles ranges from $16-30 \mathrm{~nm}$ for the $\mathrm{SE}_{1}$ combination.

The SEM, Hr-SEM and TEM images revealing the shapes and sizes of the nanoparticles formed from the $\mathrm{SE}_{2}$ combination is presented in Figure 6. It is seen that these reaction combinations resulted in anisotropic nanoparticles of triangular, pentagonal and hexagonal shapes. The sizes of the nanoparticles ranged from 25-75 $\mathrm{nm}$ for the reaction combination $\mathrm{SE}_{2}$.

The EDX spectra shows the presence of strong signal for gold atoms in the synthesized AuNPs (Insets of Figures 5 and 6). Weak signals from carbon, nitrogen and oxygen atoms are also seen which are likely to be due to X-ray emission from proteins/ enzymes present in the residual plant extracts present along with the nanoparticles. An optical absorption band at approximately $2 \mathrm{keV}$ is seen, which is characteristic of gold nanoparticles $[9,22]$.

Table 1: Extract-metal combinations studied for gold nanoparticles synthesis using T. cordifolia.

\begin{tabular}{|c|c|c|c|}
\hline \multirow{2}{*}{ Plant used } & $\begin{array}{c}\text { Plant part used } \\
\text { for preparing the } \\
\text { extract }\end{array}$ & $\begin{array}{c}\text { Concentration of component } \\
\text { in the reaction mixture } \\
\text { (mg/ L) }\end{array}$ \\
\cline { 3 - 4 } & Stem $\left(\mathrm{SE}_{1}\right)$ & 5000 & Extract \\
\hline T. cordifolia & Au (III) \\
\hline & Stem $\left(\mathrm{SE}_{2}\right)$ & 2500 & 255 \\
\hline
\end{tabular}

Table 2: Summary of UV-visible spectral studies of gold nanoparticles synthesized using T. cordifolia.

\begin{tabular}{|c|c|c|c|c|}
\hline \multicolumn{2}{|c|}{ Extract + metal ion } & \multirow{3}{*}{$\begin{array}{c}\mathrm{SE}_{1} \\
-\end{array}$} & \multicolumn{2}{|c|}{$\mathrm{SE}_{2}$} \\
\hline \multirow{2}{*}{$0^{\text {th }} \mathrm{h}$} & $\lambda_{\max }$ & & \multirow{6}{*}{\multicolumn{2}{|c|}{-}} \\
\hline & Abs. & & & \\
\hline \multirow{2}{*}{$2^{\text {nd }} \mathrm{h}$} & $\lambda_{\max }$ & 551 & & \\
\hline & Abs. & 1.566 & & \\
\hline \multirow{2}{*}{$4^{\text {th }} \mathrm{h}$} & $\lambda_{\max }$ & 551 & & \\
\hline & Abs. & 1.688 & & \\
\hline \multirow{2}{*}{$6^{\text {th }} \mathrm{h}$} & $\lambda_{\max }$ & 551 & 566 & 844 \\
\hline & Abs. & 1.697 & 1.117 & 1.089 \\
\hline \multirow{2}{*}{$24^{\text {th }} \mathrm{h}$} & $\lambda_{\max }$ & 551 & 555 & 980 \\
\hline & Abs. & 1.867 & 1.337 & 1.524 \\
\hline \multirow{2}{*}{$48^{\text {th }} \mathrm{h}$} & $\lambda_{\max }$ & 551 & 555 & 962 \\
\hline & Abs. & 1.856 & 1.260 & 1.418 \\
\hline
\end{tabular}

Table 3: $2 \theta$ position of the Bragg's plane observed from the X-ray diffractograms.

\begin{tabular}{|c|c|c|}
\hline \multirow{2}{*}{ Bragg's planet } & \multicolumn{2}{|c|}{ 20 position } \\
\cline { 2 - 3 } & SE $_{1}$ & SE $_{2}$ \\
\hline$(111)$ & 38.13 & 38.19 \\
\hline$(200)$ & 44.35 & 44.53 \\
\hline$(220)$ & 64.73 & 64.71 \\
\hline$(311)$ & 77.75 & 77.40 \\
\hline
\end{tabular}


The SAED patterns are shown in Figures 5 (h) and 6 (h) The bright circular spots correspond to the Bragg's planes representing crystalline nature of the gold nanoparticles [23]

\section{X-ray diffraction (XRD) studies}

The diffractograms (Figure 7) recorded from the powder XRD studies show intense peaks at the $2 \theta$ position, matching with (111), (200), (220) and (311) Bragg's planes and indicating a FCC (Face Centered Cubic) structure of the GNPs [24] (Table $3)$. The XRD patterns which matches with the database of JCPDS nanoparticles were calculated using the Debye-Scherrer's equation by obtaining the FWHM of the (111) Bragg's reflection from the XRD spectrum [24].

The crystallite sizes of the AuNPs were found to be ranging from 22.3 to $51.4 \mathrm{~nm}$. The ratio of optical density between the (200) and (111) Bragg's diffraction peaks were calculated to be in the range of 0.02-0.12. This is found to be lesser than the intensity ratio (i.e. 0.52 ) of conventional bulk gold, indicating the presence of nanoparticles with (111) facets [25].

\section{Fourier Transform Infra-Red (FT-IR) spectroscopy}

FT-IR analysis was used to identify the functional group of the biomolecules found in the plant extract which could have been involved in the bioreduction and capping/stabilization of the synthesized nanoparticles.

The FT-IR pattern of stem extract showed the presence of medium or strong absorption bands at 3394, 2925, 1606, 1388, and $1037 \mathrm{~cm}^{-1}$. The strongest absorption band can be assigned to $-\mathrm{NO}_{2}$ (at 1606 and $1388 \mathrm{~cm}^{-1}$ ) of aliphatic nitro group. Bands originating from hydroxyl group (free water and/or alcohols) at $\sim 3300$ and $1080 \mathrm{~cm}^{-1}$, as well as a medium band at $1606 \mathrm{~cm}^{-1}$, indicated the presence of amide/amine groups. Comparison of the spectra of stem extract (Figure 8a) and gold nanoparticles (Figure $8 \mathrm{~b}$ ) reveals minor changes in the positions as well as on the magnitude of the absorption bands. On closer examination, the spectrum of gold nanoparticles shows bands at 1643 and $1521 \mathrm{~cm}^{-1}$ (due to $\mathrm{N}-\mathrm{H}$ bond) confirming the presence of amines. As presence of alkaloids in T. cordifolia stem has been reported, [26-28] it is possible that the amino groups are a part of those alkaloids.

\section{Mechanism of AuNPs formation}

It is generally accepted $[6-8,14]$ that a 2 -step mechanism operates in the generation of nanoparticles when trivalent $\mathrm{Au}$ ions are mixed with aqueous extracts of plants: a) reduction of the gold ions to zerovalent gold is caused by the biomolecules

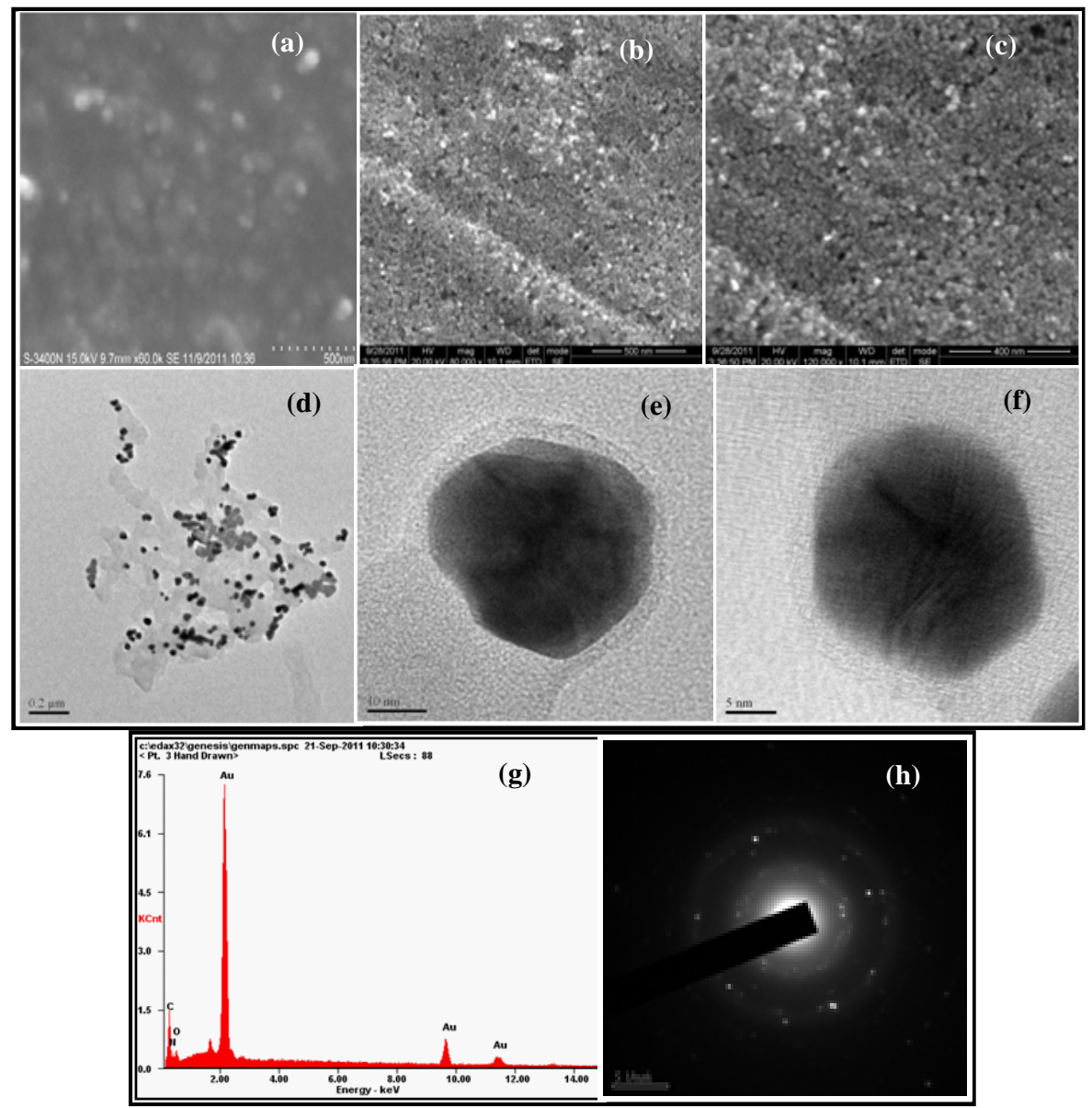

Figure 5: Each visual is a composite of (a) scanning electron micrograph; (b) \& (c) high resolution electron micrographs; (d), (e) \& (f) transmission electron micrographs; (g) EDX spectrum; (h) SAED pattern of gold nanoparticles synthesized using T. cordifolia (SE). 

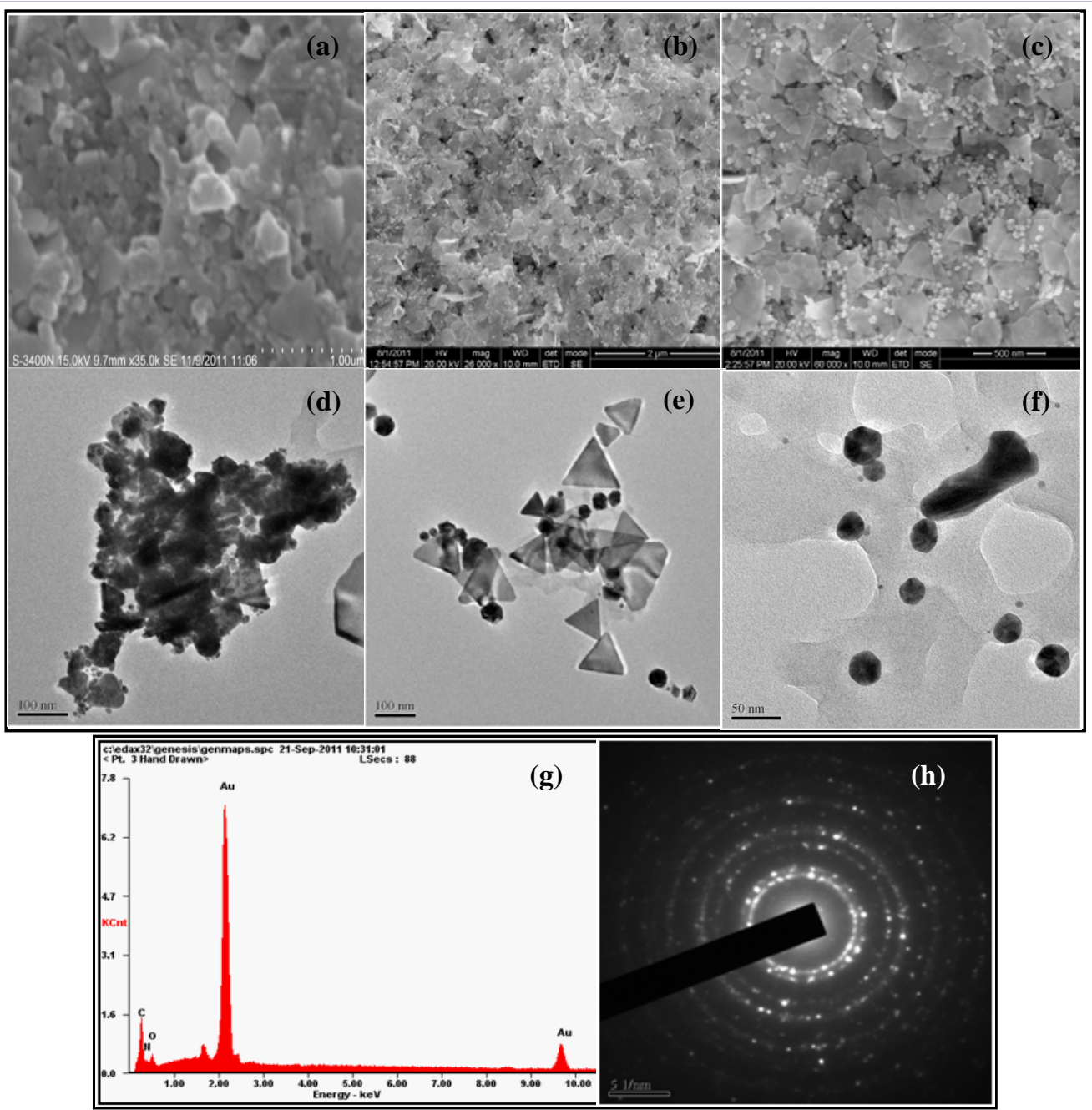

Figure 6: Each visual is a composite of (a) scanning electron micrograph; (b) \& (c) high resolution electron micrographs; (d), (e) \& (f) transmission electron micrographs; (g) EDX spectrum; (h) SAED pattern of gold nanoparticles synthesized using T. cordifolia $\left(\mathrm{SE}_{2}\right)$.
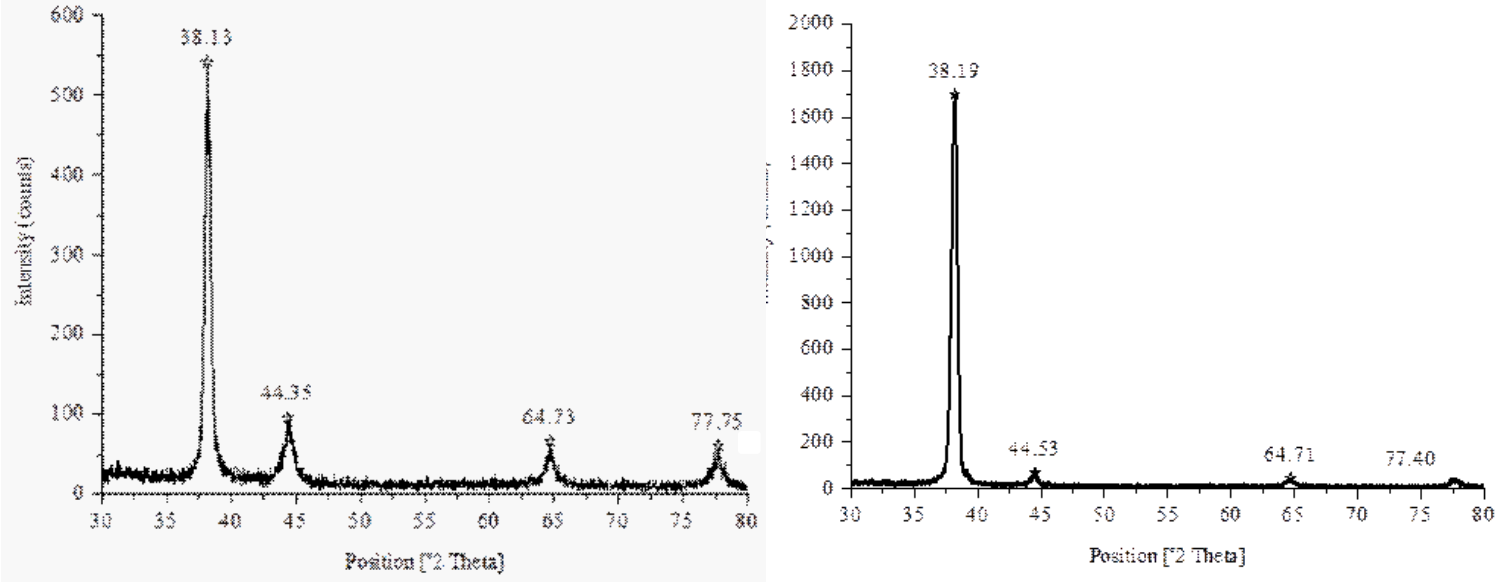

Figure 7: X-ray diffraction spectrum of (a) $\mathrm{SE}_{1}$ and (b) $\mathrm{SE}_{2}$ gold nanoparticles.

Citation: Abbasi T, Anuradha J, Abbasi SA (2014) Utilization of the Terrestrial Weed Guduchi (Tinospora cordifolia) in Clean-Green

Synthesis of Gold Nanoparticles. Nanosci Technol 1(3): 1-7. 
present in the plant extract and, b) agglomerating gold atoms are stabilized at nano-size by the enveloping of the biomolecules around them (Figure 9). The same mechanism is likely to have been operative in case of AuNPs formed when T. cordifolia was employed.

\section{Conclusion}

1. The studies reveal that the stem extracts of the weed guduchi (Tinospora cordifolia) have the potential to reduce $\mathrm{Au}$ (iii) ions to gold nanoparticles (AuNPs) and also stabilize them.

2. It was seen that by varying the concentration of plant extract relative to gold (iii) solution, different sizes and shapes of AuNPs can be generated, ranging from isotropic spherical to anisotropic triangular, hexagonal and pentagonal.

3. The sizes of the isotropic AuNPs, as revealed by electron microscopic and X-ray diffraction studies, ranged from $16 \mathrm{~nm}$ to $30 \mathrm{~nm}$ and that of anisotropic ranged from 25 to $75 \mathrm{~nm}$. Hence the process is capable of generating nanoparticles within a wide range of shapes and sizes.

4. FT-IR spectral studies indicated that alkaloids might be playing a major role in the reduction of the trivalent gold to zero-valent AuNPs and the subsequent stabilization/ capping of the resulting AuNPs.

\section{Acknowledgement}

Authors thank the University Grants Commission, New Delhi, for support.
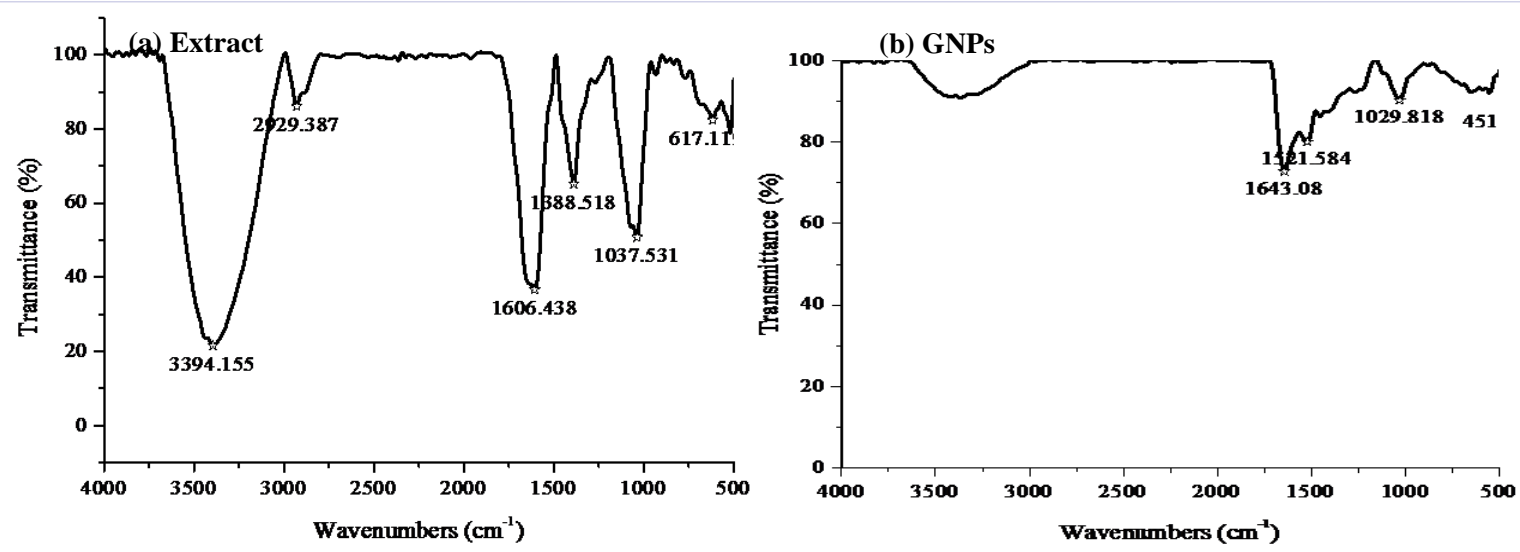

Figure 8: The FTIR spectra of T. cordifolia stem extract and of the synthesized gold nanoparticles.

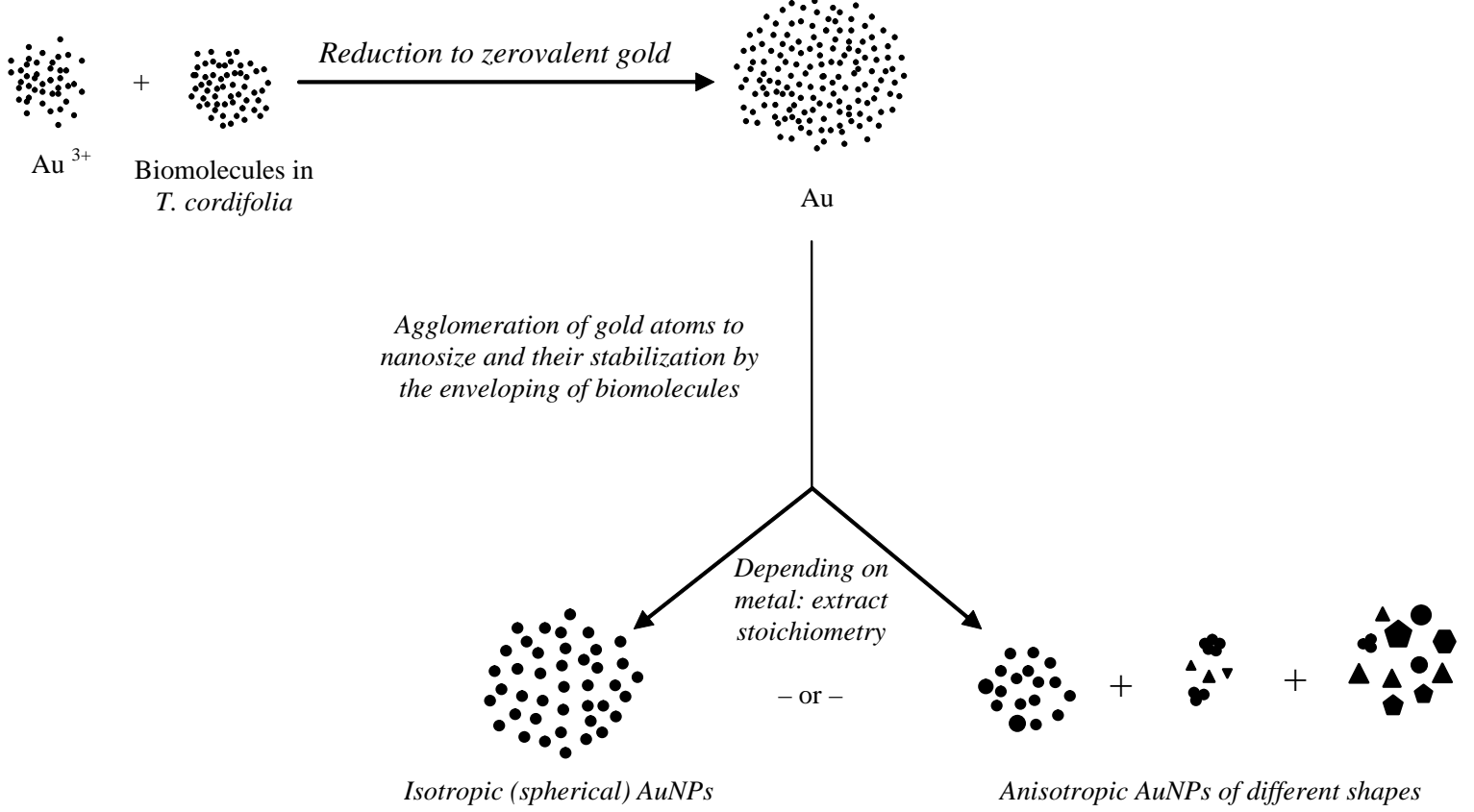

Figure 9: Likely mechanism of the formation of isotropic and anisotropic AuNPs by the action of T. cordifolia on trivalent gold. 


\section{References}

1. Anuradha J, Abbasi T, AbbasiSA. 'Green' synthesis of gold nanoparticles with aqueous extracts of neem (Azadirachta indica). Research J of Biotech. 2010; 5(1): 75-79.

2. Abbasi SA, Abbasi T, Anuradha J. A process for synthesis of metal nanoparticles from aquatic weeds. Offl J Patent Off. 2012; 6184.

3. Abbasi SA, Abbasi T, Neghi N, Ganaie SU. Gainful utilization of four otherwise worthless and problematic weeds for silver nanoparticle synthesis. Offl J Patent Off. 2011; 11869.

4. Anuradha J, Abbasi T, Abbasi SA, Biomimetic. Synthesis of gold nanoparticles using Aloe vera. Inter J Environ Sci \& Engi. Res. 2011; 2: $1-5$.

5. APHA (American Public Health Association), wastewater. 22nd ed. American Public Health Association, American Water Works Association and Water Environment Federation publication. Washington DC (Standard methods of water and USA) 2012.

6. Abbasi T, Anuradha J, Abbasi SA. Use of the plants in biomimetic synthesis of gold nanoparticles. Journal of Nanoscience, in press. 2014.

7. Abbasi T, Anuradha J, Ganaie SU, Abbasi SA. Gainful utilization of the highly intransigent weed ipomoea in the synthesis of gold nanoparticles. Journal of King Saud University. Science. 2014. doi: 10.1016/j.jksus.2014.04.001

8. Borchert H, Shevchenko EV, Robert A, Mekis I, Kornowski A, Grubel G, et al. Determination of nanocrystal sizes: a comparison of TEM, SAXS, and XRD studies of highly monodisperse CoPt3 particles. Langmuir. 2005; 21(5): 1931-1936.

9. Chauhan K. Successful treatment of throat cancer with ayurvedic drugs. Suchitra Ayurved. 1995; 47: 840-842.

10.Deshpande R, Bedre DM, Basavaraja S, Sawle B, Manjunath SY, Venkataraman A. Rapid biosynthesis of irregular shaped gold nanoparticles from macerated aqueous extracellular dried clove buds (Syzygium aromaticum) solution. Coll. Surf. B Biointerfaces. 2010; 79(1): 235-240. doi: 10.1016/j.colsurfb.2010.04.003.

11. Ghosh P, Han G, De M, Kim CK, Rotello VM. Gold nanoparticles in delivery applications. Adv. Drug Deliver. Rev. 2008; 60(11): 13071315. doi: 10.1016/j.addr.2008.03.016.

12. Ganaie SU, Abbasi T, Anuradha J, Abbasi SA. Biomimetic synthesis of silver nanoparticles using the amphibious weed ipomoea and their application in pollution control. Journal of King Saud UniversityScience. 2014; 26(3): 222-229. doi: 10.1016/j.jksus.2014.02.004.

13. Jayaseelan C, Rahuman AA, Rajakumar G, Kirthi AV, Santhoshkumar T, Marimuthu S. Synthesis of pediculocidal and larvicidal silver nanoparticles by leaf extract from heartleaf moonseed plant, Tinospora cordifolia Miers. Parasitol Res. 2011; 109: 185-194. doi: 10.1007/s00436-010-2242-y.

14. Khare V, Li Z, Mantion A, Ayi AA, Sonkaria S, Voelkl A. Strong anion effects on gold nanoparticle formation in ionic liquids. J. Mater. Chem. 2010; 20(7): 1332-1339. doi: 10.1039/B917467B.
15. Link S, El-Sayed MA. Optical properties and ultrafast dynamics of metallic nanocrystals. Annu. Re. Phys. Chem. 2003; 54: 331-366. doi: 10.1146/annurev.physchem.54.011002.103759.

16. Liu S, Leech D, Ju H. Application of colloidal gold in protein immobilization, electron transfer, and biosensing. Anal. Lett. 2003; 36(1): 1-19. doi: 10.1081/AL-120017740.

17. Liz-Marzan, LM. Tailoring surface plasmons through the morphology and assembly of metal nanoparticles. Langmuir. 2006; 22(1): 32-41.

18. Morones JR, Elechiguerra JL, Camacho A, Holt K, Kouri JB, Ramfrez JT, et al. The bactericidal effect of silver nanoparticles. Nanotechnology. 2005; 16(10): 2346-2353.

19. Mulvaney P. Surface Plasmon Spectroscopy of nanosized metal particles. Langmuir. 1996; 12(3): 788-800. doi: 10.1021/la9502711.

20. Philip D. Biosynthesis of $\mathrm{Au}, \mathrm{Ag}$ and $\mathrm{Au}-\mathrm{Ag}$ nanoparticles using edible mushroom extract. Spectrochimica Acta Part A. 2009; 73(2): 374-381. doi: 10.1016/j.saa.2009.02.037.

21. Prakash NKU, Bhuvaneswari S, Jahnavi B, Abhinaya K, Rajalin AG, Kumar MP, et al. A study on antibacterial activity of common weeds in Northern districts of Tamil Nadu, India. Research Journal of Madical Plant. 2012; 6(4): 341-345. doi: 10.3923/rjmp.2012.341.345.

22. Raghunandan D, Bedre MD, Basavaraja S, Sawle B, Manjunath SY, Venkataraman A. Rapid biosynthesis of irregular shaped gold nanoparticles from macerated aqueous extracellular dried clove buds (Syzygium aromaticum) solution. Colloid. Surface. B. 2010; 79(1): 235-240. doi: 10.1016/j.colsurfb.

23. Rajathi K, Raj DV, Anarkali J, Sridhar S. Green synthesis characterization and in-vitro antibacterial activity of silver nanoparticles by using Tinospora cordifolia leaf extract. Inter. J. Green Chemistry and Bioprocess. 2012; 2(2): 15-19.

24. Shankar SS, Ahmad A, Parsricha R, Sastry M. Bioreduction of chloroaurate ions by geranium leaves and its endophytic fungus yields gold nanoparticles of different shapes. J. Mat. Chem. 2003; 13(7): 1822-1826. doi: 10.1039/B303808B.

25. Shankar SS, Rai A, Ahmad A, Sastry M. Controlling the optical properties of lemongrass extract synthesized gold nanotriangles and potential application in infrared-absorbing optical coatings. Chem. Mater. 2005; 17(3): 566-572. doi: 10.1021/cm048292g.

26. Singh SS, Pandey SC, Srivastava S, Gupta VS, Patro B, Ghosh AC. Chemistry and medicinal properties of Tinospora cordifolia (Guduchi). Indian J. Pharmacology. 2003; 35: 83-91.

27. Tiwari PM, Vig K, Dennis VA, Singh SR. Functionalized gold nanoparticles and their biomedical applications. Nanomaterials. 2011; 1(1): 31-63. doi: 10.3390/nano1010031.

28. Wen B, Ma J, Chen C, Ma W, Zhu H, Zhao J. Supported noble metal nanoparticles as photo/sono-catalysts for synthesis of chemicals and degradation of pollutants. Sci. China Chem. 2011; 54(6): 887-897. 